\title{
Higgs Bosons and the Indirect Search for WIMPs
}

\author{
V. A. Bednyakov ${ }^{2}$, H. V. Klapdor-Kleingrothaus ${ }^{1}$, and $\mathrm{H}$. $\mathrm{Tu}^{1}$ \\ 1 Max-Planck-Institut für Kernphysik, Postfach 103980, D-69029 Heidelberg, \\ Germany \\ ${ }^{2}$ Laboratory of Nuclear Problems, Joint Institute for Nuclear Research, Moscow \\ region, 141980 Dubna, Russia
}

\begin{abstract}
We investigated the contribution of the MSSM Higgs bosons produced in the neutralino annihilation in the Earth and Sun to the total WIMPs detection signal. We found that this contribution is very important and results in a lower bound for the muon flux from the Sun of $10^{-7} \div 10^{-8} \mathrm{~m}^{-2} \mathrm{yr}^{-1}$ for neutralinos heavier than about $200 \mathrm{GeV}$. We noticed that due to the SUSY charged Higgs bosons one can expect an energetic $\tau$ neutrino flux from the Sun at a level of $10^{2} \mathrm{~m}^{-2} \mathrm{yr}^{-1}$.
\end{abstract}

\section{Introduction}

The Weakly-Interacting Massive Particles (WIMPs) as the most attractive and well-motivated cold dark-matter candidates in the Galactic Halo may be gravitationally trapped by astrophysical objects like the Earth or the Sun due to subsequent energy losses via elastic scatterings off the nuclei therein. The trapped WIMPs would undergo pair annihilations in the core of the Earth or the Sun, producing all kinds of ordinary particles like quarks, leptons, gauge and Higgs bosons. The neutrinos produced in the decays of these WIMP annihilation products provide detectable signals, because among all particles from WIMPs annihilation only neutrinos can pass through the astrophysical bodies and reach the detectors. The flux of the neutrinos of type $i$ (e.g. $i=\nu_{\mu}, \bar{\nu}_{\mu}$, or $\nu_{\tau}, \bar{\nu}_{\tau}$ ) from WIMP annihilation in the Earth or Sun is [1]

$$
\left(\frac{d \phi}{d E}\right)_{i}=\frac{\Gamma_{A}}{4 \pi R^{2}} \sum_{F} B_{F}\left(\frac{d N}{d E}\right)_{F, i}
$$

where $\Gamma_{A}$ is the annihilation rate of the WIMPs in the Sun or Earth, $R$ is the distance the neutrinos have to travel to the detector, $B_{F}$ is the branching ratio for annihilation into final state $F$, and $(d N / d E)_{F, i}$ is the differential energy spectrum of the $i$-type neutrinos at the surface of the Sun or Earth expected from decays of particles produced in channel $F$ in the core of the Sun or Earth. Recent information about indirect WIMP detection can be found in e.g. [1,2].3, , 4 , or in the talks given by T. Montaruli, O. Suvorova, J. A. Djilkibaev, N. Fornengo, A. Bottino at this conference.

Among all WIMPs, the neutralinos are the most considered, which arise from the supersymmetric extensions of the Standard Model and are most likely the 
Lightest Supersymmetric Particle (LSP). In SUSY scenarios with large sfermion and gaugino masses even the LSP should also be heavy and thus cannot be produced at current or near-future colliders. In this case the Higgs bosons still have chances to be light and specifically the detection of the charged Higgs boson could serve as first evidence for supersymmetry.

With this interest in the Higgs bosons, in this work we perform an investigation within the framework of the Minimal Supersymmetric extension of the Standard Model on the Higgs bosons produced in the neutralino annihilation in the Earth and Sun and their role in the detection of the WIMPs. Following our approach in [5], we relax any constraints from unification assumptions on the parameters. On the other hand we use the results from collider searches for supersymmetric particles, rare FCNC $b \rightarrow s \gamma$ decay, as well as bounds on the WIMPs relic abundances to constrain the MSSM parameter space. Our free parameters are $\tan \beta, \mu, M_{1}, M_{2}, M_{A}, m_{\tilde{Q}}^{2}, m_{\tilde{L}}^{2}, m_{\tilde{Q}_{3}}^{2}, m_{\tilde{L_{3}}}^{2}$ and $A_{t}$ (see [5]).

\section{Higgs Boson Contribution to Indirect WIMP Search}

The Minimal Supersymmetric extension of the Standard Model (MSSM) possesses two complex scalar doublets, after the spontaneous electroweak-symmetry breaking five physical Higgs bosons appear in the MSSM particle spectrum. Assuming $C P$ invariance, the MSSM Higgs sector contains a neutral $C P$-odd Higgs boson $A^{0}$, two neutral $C P$-even Higgs bosons $H^{0}, h^{0}$, which are mixtures of the neutral Higgs interaction eigenstates, and a pair of charged Higgs bosons $H^{ \pm}$. All the five MSSM Higgs bosons can be produced in neutralino pair annihilation processes, either in pairs or accompanied by a gauge boson. However, since the pair annihilation of the neutralinos captured in the Sun and Earth takes place practically at rest, only processes allowed by $C P$ conservation in the $v \rightarrow 0$ limit are relevant for the indirect WIMPs detection:

$$
\chi \chi \rightarrow h^{0} Z^{0}, h^{0} A^{0}, H^{0} Z^{0}, H^{0} A^{0}, W^{ \pm} H^{\mp} .
$$

The Higgs bosons produced in neutralino annihilation decay immediately before losing energy. Because the Higgs couplings to fermion pairs are proportional to the fermion masses (see for example [6]), the neutral Higgs bosons do not decay into neutrinos directly, and the branching ratio for charged Higgs boson decay into muon neutrino is negligible. Also, for the Higgs bosons produced in the LSP neutralino annihilation at rest the decay into superpartners is kinematically forbidden. Therefore we consider the following two-body decay channels of the five MSSM Higgs bosons which can produce energetic muon neutrinos

$$
\begin{aligned}
& h^{0} \rightarrow \tau \bar{\tau}, b \bar{b}, c \bar{c}, t \bar{t}, W^{+} W^{-}, Z^{0} Z^{0}, Z^{0} \gamma \\
& H^{0} \rightarrow \tau \bar{\tau}, b \bar{b}, c \bar{c}, t \bar{t}, W^{+} W^{-}, Z^{0} Z^{0}, Z^{0} \gamma, Z^{0} A^{0}, h^{0} h^{0}, A^{0} A^{0}, W^{ \pm} H^{\mp} ; \\
& A^{0} \rightarrow \tau \bar{\tau}, b \bar{b}, c \bar{c}, t \bar{t}, Z^{0} \gamma, Z^{0} h^{0} ; \\
& H^{+} \rightarrow \tau^{+} \nu_{\tau}, c \bar{b}, c \bar{s}, t \bar{b}, h^{0} W^{+}, A^{0} W^{+}+\text {charge conjugation. }
\end{aligned}
$$


The decay branching ratios for the above channels are calculated with the computer code HDECAY written by A. Djouadi et al. [7], in which all relevant higher-order QCD corrections to decays into quarks and gluons, all important below-threshold three-body decays, and complete radiative corrections in the effective potential approach are included.

We investigate the Higgs boson contribution to the WIMPs detection signal (11) by switching off the final states containing Higgs bosons in our calculation (that is, we do not count the neutrinos from these channels) and compare the results $\phi_{\text {noHiggs }}$ with that from all channels including Higgs final states $\phi_{\text {withHiggs }}$, which is calculated using the formulae given in [1].
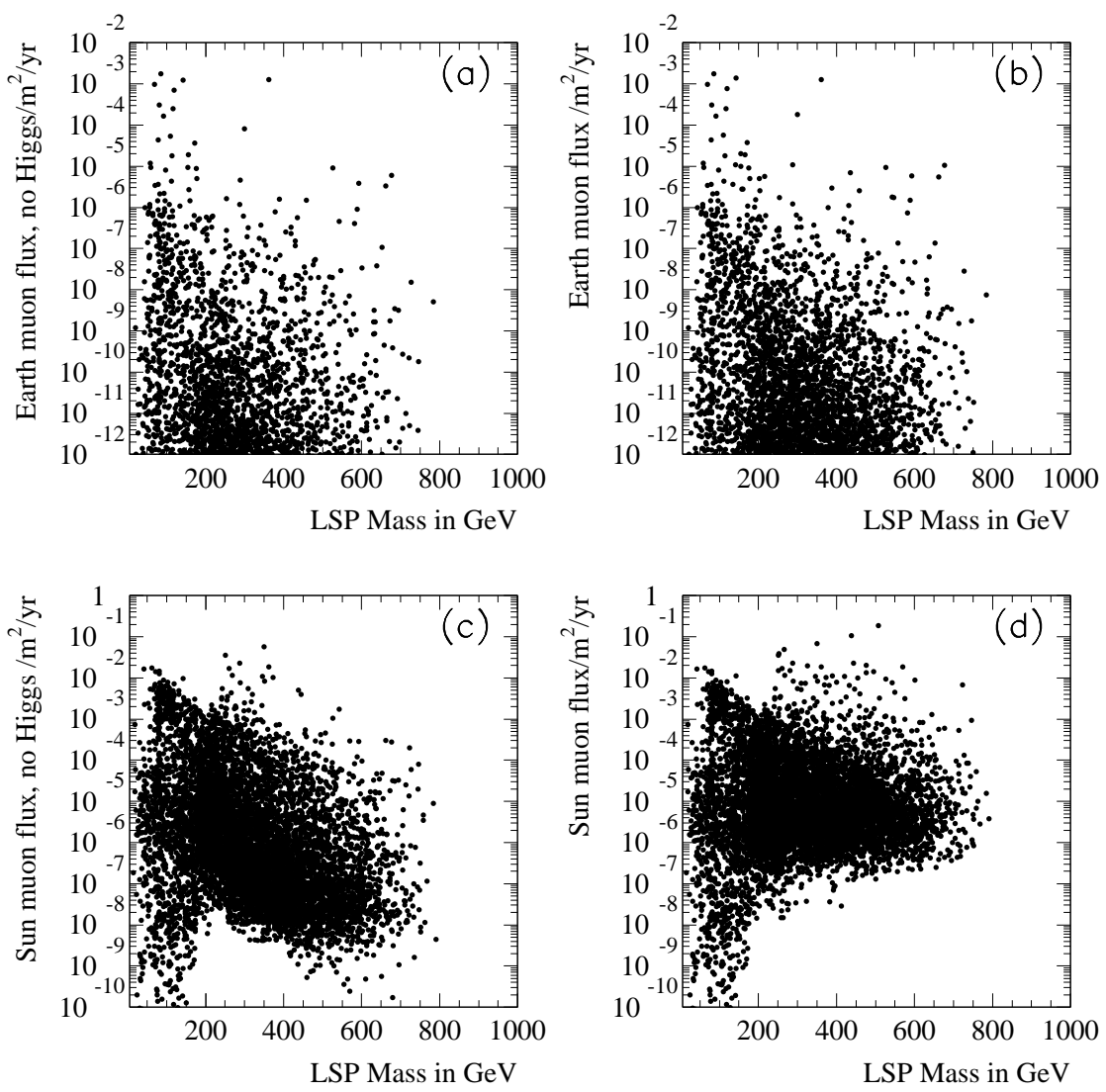

Fig. 1. Upward-going muon detection rate from the Earth (a) without contribution from final states containing Higgs bosons $\phi_{\text {noHiggs }}^{\oplus}$ and (b) with all channels including Higgs final states $\phi_{\text {withHiggs }}^{\oplus}$ as well as from the Sun (c) without $\phi_{\text {noHiggs }}^{\odot}$, (d) with $\phi_{\text {withHiggs }}^{\odot}$ Higgs final states. 
In figure 1 we plot our results for $\phi_{\text {noHiggs }}$ and $\phi_{\text {withHiggs }}$ versus the neutralino mass $m_{\chi}$. Comparing figure 11(c) with 11(d) we see (it can be seen more easily in the case of the Sun), that there are significant differences between them mainly in the region where the total detection rate for upward-going muons induced by neutrinos from the Sun is $10^{-7} \div 10^{-9} \mathrm{~m}^{-2} \mathrm{yr}^{-1}$ for neutralinos heavier than about $200 \mathrm{GeV}$. In order to see the Higgs final state contribution quantitatively we plot in figure 2 the ratio of the two detection rates $\phi_{\text {noHiggs }} / \phi_{\text {withHiggs }}(\mathrm{a}),(\mathrm{b})$ versus neutralino mass $m_{\chi}$ and (c), (d) versus the upward-going muon detection rate with contribution from all channels $\phi_{\text {all }}$.
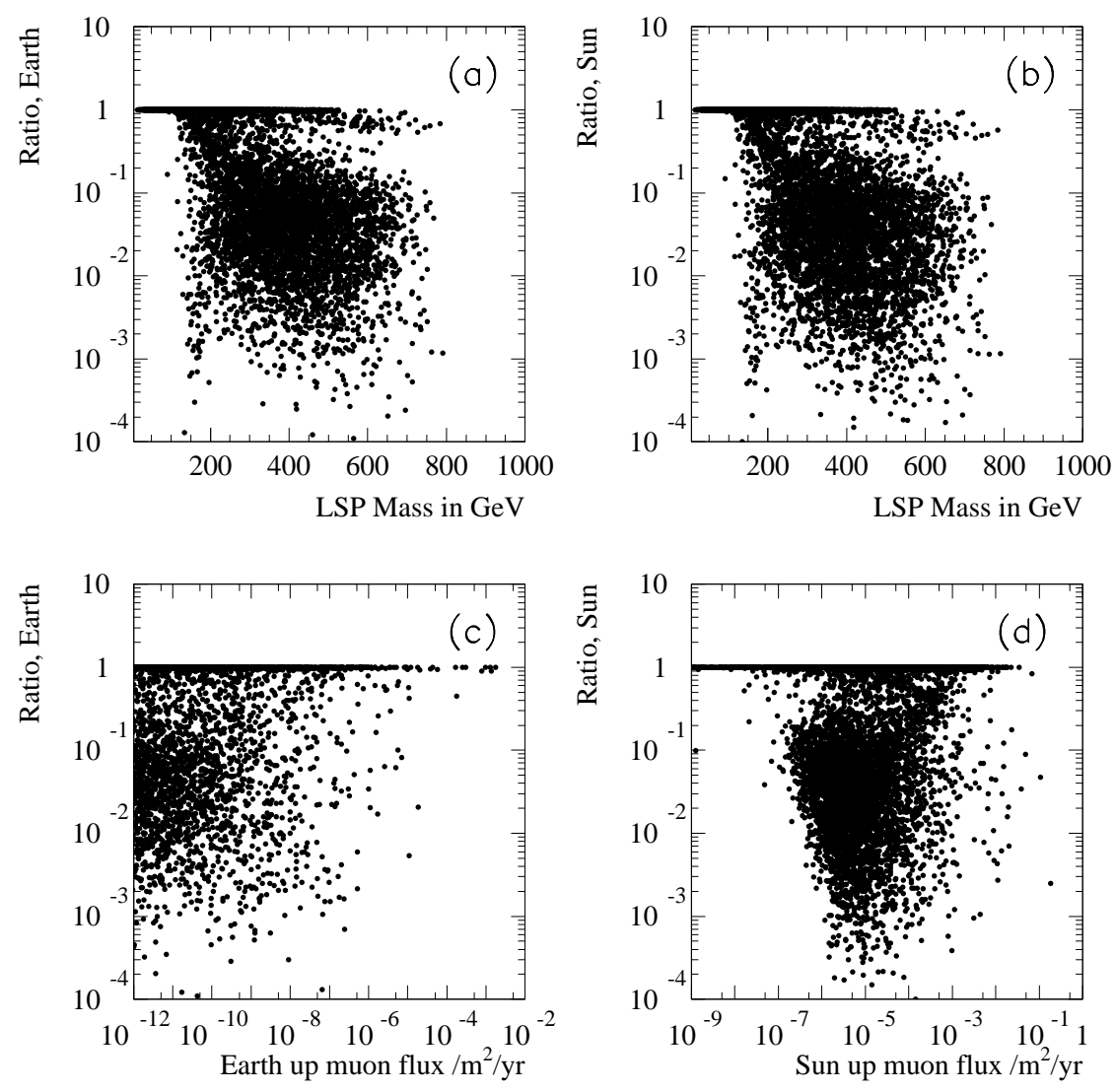

Fig. 2. Ratio of the detection rate without Higgs final states to from all channels including Higgs final states (a) and (b) versus $m_{\chi},(\mathrm{c})$ and (d) versus the detection rate $\phi_{\text {withHiggs }}$.

In figure 2(a) and 2(b) it can be seen that for neutralinos heavier than 200 $\mathrm{GeV}$ the Higgs final states are in general important. If their contributions were not included the indirect WIMP detection rate could be roughly $10^{1}$ to $10^{3}$ 
times lower. Figure 2(d) shows that in the case of the Sun, the Higgs final state contribution is important in the region $10^{-7} \leq \phi_{\text {all }}^{\odot} \leq 10^{-3} \mathrm{~m}^{-2} \mathrm{yr}^{-1}$ where one should expect the signals.

The Higgs contribution is not important in the region where the upwardgoing muon detection rate from the Sun is lower than $10^{-7} \mathrm{~m}^{-2} \mathrm{yr}^{-1}$, because such low detection rates are expected mainly for neutralinos lighter than about $200 \mathrm{GeV}$ (see figure 1(d)). In this case the neutralino annihilation into final states containing Higgs bosons may have chances to compete with, or even dominate over the fermion and/or gauge boson pair final states if kinematically allowed, but since the Higgs bosons do not produce neutrinos directly, the neutrinos from the Higgses are not so energetic compared to those directly produced in the fermion or gauge boson decay. As a consequence, their contribution to the total detection rate which is proportional to the product of their branching ratios $B_{F}$ and the second moment of the neutrino spectra $(d N / d E)_{F, i}$, is not important for lighter neutralinos.

The indirect dark matter search experiments have now reached a sensitivity of $\sim 10^{-3} \mathrm{~m}^{-2} \mathrm{yr}^{-1}$, so our conclusion is that the Higgs bosons are important as far as the current indirect dark matter search experiments are concerned. The contribution from final states containing Higgs bosons results in a lower bound for the muon flux from the Sun of $10^{-7} \div 10^{-8} \mathrm{~m}^{-2} \mathrm{yr}^{-1}$ for neutralinos heavier than about $200 \mathrm{GeV}$. In other words, if the collider experiments show that the neutralino is so heavy, one should see signals at the latest as the indirect search experiments reach a sensitivity of about $10^{-7} \mathrm{~m}^{-2} \mathrm{yr}^{-1}$. And this is due to the contribution from the Higgs final states.

\section{Energetic Tau Neutrinos from Charged Higgs Boson}

In the last section we saw that the charged Higgs boson can decay directly into $\tau$ neutrinos via $H^{+} \rightarrow \tau^{+} \nu_{\tau}$, which is the dominant channel below the $t b$ threshold in most cases except for small $\tan \beta$ values.

The $\tau$ neutrinos as the direct decay product of the charged Higgs from neutralino annihilation can be expected to have the highest energies among all $\tau$ neutrinos from WIMPs and have a harder energy spectrum. The charged Higgs boson produced in $\chi \chi \rightarrow W^{ \pm} H^{\mp}$ decays with energy between $m_{\chi}$ and $2 m_{\chi}$ depending on the charged Higgs mass (currently we know that $m_{\mathrm{Ch}} \geq 78.6 \mathrm{GeV}$ [8]). The maximum energy the $\tau$ neutrinos can obtain from the charged Higgs boson produced in neutralino annihilation is always about the neutralino mass $m_{\chi}$, independent of the charged Higgs mass $m_{\mathrm{Ch}}$. The channel $\chi \chi \rightarrow W^{ \pm} H^{\mp}$ is open when the kinematic condition $2 m_{\chi}>m_{W}+m_{\mathrm{Ch}}$ is satisfied. One thing interesting is that when the charged Higgs mass $m_{\mathrm{Ch}}$ not deviates too much from $2 m_{\chi}-m_{W}$, the $\tau$ neutrino energy spectrum should have the form of a relatively sharp peak at half the energy of the decaying charged Higgs 1 . In contrast, other neutralino annihilation products like fermion and gauge boson pairs

\footnotetext{
${ }_{1}$ Indeed, if one assumes $M_{W} \ll m_{\mathrm{Ch}}$ (or equivalently $M_{W} \approx 0$ ), after the annihilation $\chi \chi \rightarrow W^{ \pm} H^{\mp}$ both $W$ and Higgs bosons can appear at rest and $E_{H^{+}}=m_{\mathrm{Ch}} \approx 2 m_{\chi}$.
} 
decay always with energy equal to $m_{\chi}$ and generate a much flatter $\tau$ neutrino spectrum.

The flux of the $\tau$ neutrinos from the charged Higgs bosons produced in WIMPs annihilation and decaying with energy and velocity $E_{\mathrm{Ch}}$ and $\beta_{\mathrm{Ch}}$, respectively, can be calculated using the formulae for gauge boson decays given in [9]

$$
\begin{aligned}
&\left(\frac{d \phi}{d E}\right)_{H^{+}}^{\oplus}\left(E_{\nu}\right)= \frac{\Gamma_{A}}{4 \pi R^{2}} \operatorname{Br}\left(\chi \chi \rightarrow H^{ \pm} W^{\mp}\right)\left(\frac{d N}{d E}\right)_{H^{+}}\left(E_{\nu}\right) \\
&=\frac{\Gamma_{A}}{4 \pi R^{2}} \operatorname{Br}\left(\chi \chi \rightarrow H^{ \pm} W^{\mp}\right) \frac{\Gamma\left(H^{+} \rightarrow \tau^{+} \nu_{\tau}\right)}{E_{\mathrm{Ch}} \beta_{\mathrm{Ch}}} \times \\
& \Theta\left(E_{\text {low }}^{\oplus}<E_{\nu}<E_{\text {upp }}^{\oplus}\right),
\end{aligned}
$$

where

$$
E_{\text {low }}^{\oplus}=\frac{E_{\mathrm{Ch}}}{2}\left(1-\beta_{\mathrm{Ch}}\right), \quad E_{\text {upp }}^{\oplus}=\frac{E_{\mathrm{Ch}}}{2}\left(1+\beta_{\mathrm{Ch}}\right)
$$

are the lower and upper boundaries for the $\tau$ neutrino energy. $\Theta(x)$ is the Heaviside step function, $\Theta(x)=1$ if $x$ is true and 0 otherwise.

In figure 3 we plot the differential $\tau$ neutrino flux from charged Higgs boson decay in the Earth versus the maximum $\tau$ neutrino energy $E_{\text {upp }}^{\oplus}$; we also integrate the differential neutrino flux (5) over $E_{\nu}$ to obtain the total flux of the $\tau$ neutrinos from charged Higgs decay in the Earth and plot it versus the neutralino mass. The dependences of the maximum and mean energy of these $\tau$ neutrinos on the neutralino mass are also shown. The maximum energy a $\tau$ neutrino can obtain from the neutralino annihilation via charged Higgs decay $E_{\text {upp }}^{\oplus}$ is roughly the neutralino mass, as expected.

For the $\tau$ neutrinos produced in the core of the Sun, the neutrino interactions with the solar medium play an important role in determining the $\tau$ neutrino spectrum at the Sun's surface. We make a rough estimate for this effect following 10

$$
\begin{aligned}
&\left(\frac{d \phi}{d E}\right)_{H^{+}}^{\odot}\left(E_{\nu}\right)=\frac{\Gamma_{A}}{4 \pi R^{2}} \operatorname{Br}(\chi \chi\left.\rightarrow H^{ \pm} W^{\mp}\right) \frac{\Gamma\left(H^{+} \rightarrow \tau^{+} \nu_{\tau}\right)}{E_{\mathrm{Ch}} \beta_{\mathrm{Ch}}} \times \\
&\left(1-E_{\nu} \tau_{\nu}\right)^{\alpha_{\nu}-2} \Theta\left(E_{\text {low }}^{\odot}<E_{\nu}<E_{\mathrm{upp}}^{\odot}\right)
\end{aligned}
$$

with

$$
E_{\text {low }}^{\odot} \equiv \frac{E_{\text {low }}^{\oplus}}{1+E_{\text {low }}^{\oplus} \tau_{\nu}}, \quad E_{\text {upp }}^{\odot} \equiv \frac{E_{\text {upp }}^{\oplus}}{1+E_{\mathrm{upp}}^{\oplus} \tau_{\nu}}
$$

the lower and upper boundaries for the energy of the $\tau$ neutrinos from the Sun. The coefficients $\tau_{\nu_{\tau}}, \tau_{\bar{\nu}_{\tau}}, \alpha_{\nu_{\tau}}$ and $\alpha_{\bar{\nu}_{\tau}}$ are used to parametrize the neutrino

Therefore the decay $H^{+} \rightarrow \tau^{+} \nu_{\tau}$ gives $E_{\nu}=E_{\tau}=m_{\mathrm{Ch}} / 2=m_{\chi}$. Under the same assumption the competitive annihilation $\chi \chi \rightarrow W^{ \pm} W^{\mp}$ with $W \rightarrow \tau \nu$ gives $E_{W^{ \pm}}=$ $m_{\chi}$ followed by $E_{\nu}=E_{\tau}=m_{\chi} / 2$. Therefore the hardest part of the $\nu_{\tau}$ energy spectrum one can connect with the decay of the (very) heavy charged Higgs boson. 

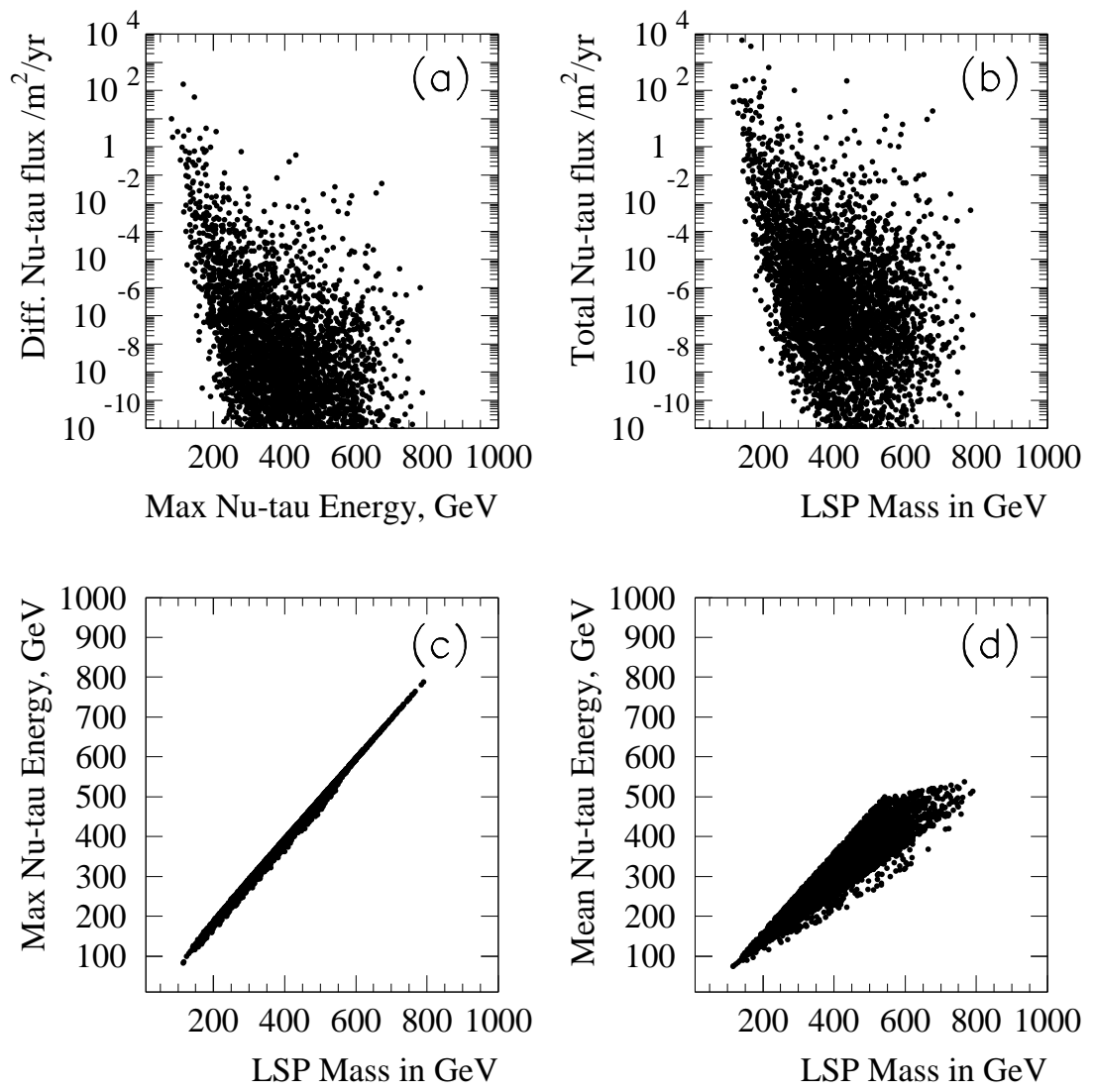

Fig. 3. (a) Differential flux of $\tau$ neutrinos from charged Higgs boson decay vs. maximum $\tau$ neutrino energy. (b) Total flux of $\tau$ neutrinos from charged Higgs bosons decay vs. neutralino mass $m_{\chi}$. (c) Maximum $\tau$ neutrino energy vs. neutralino mass $m_{\chi}$. (d) Mean $\tau$ neutrino energy vs. neutralino mass $m_{\chi}$. All for the case of the Earth.

stopping and absorption effects in the Sun 10,11. Due to the interactions with the solar medium, the $\tau$ neutrino differential flux from the Sun depends on the neutrino energy $E_{\nu}$ explicitly. We choose to plot $(d \phi / d E)^{\odot}$ versus $E_{\mathrm{upp}}^{\odot}$, since the flux is largest for the highest energy.

In figure 1 we show our results for the $\tau$ neutrinos from the charged Higgs decay in the Sun. By comparing it with figure 3 we see that the energy of the $\tau$ neutrinos from the Sun is to a large extent degraded due to the interactions with the solar medium, however, since all neutrinos produced by the neutralino annihilation in the core of the Sun suffer from these effects while propagating through the Sun, the $\tau$ neutrinos from charged Higgs decay are still the most energetic among all neutrinos from neutralino annihilation in the Sun. Furthermore, we can expect a much larger $\tau$ neutrino flux from the Sun than from the Earth. 

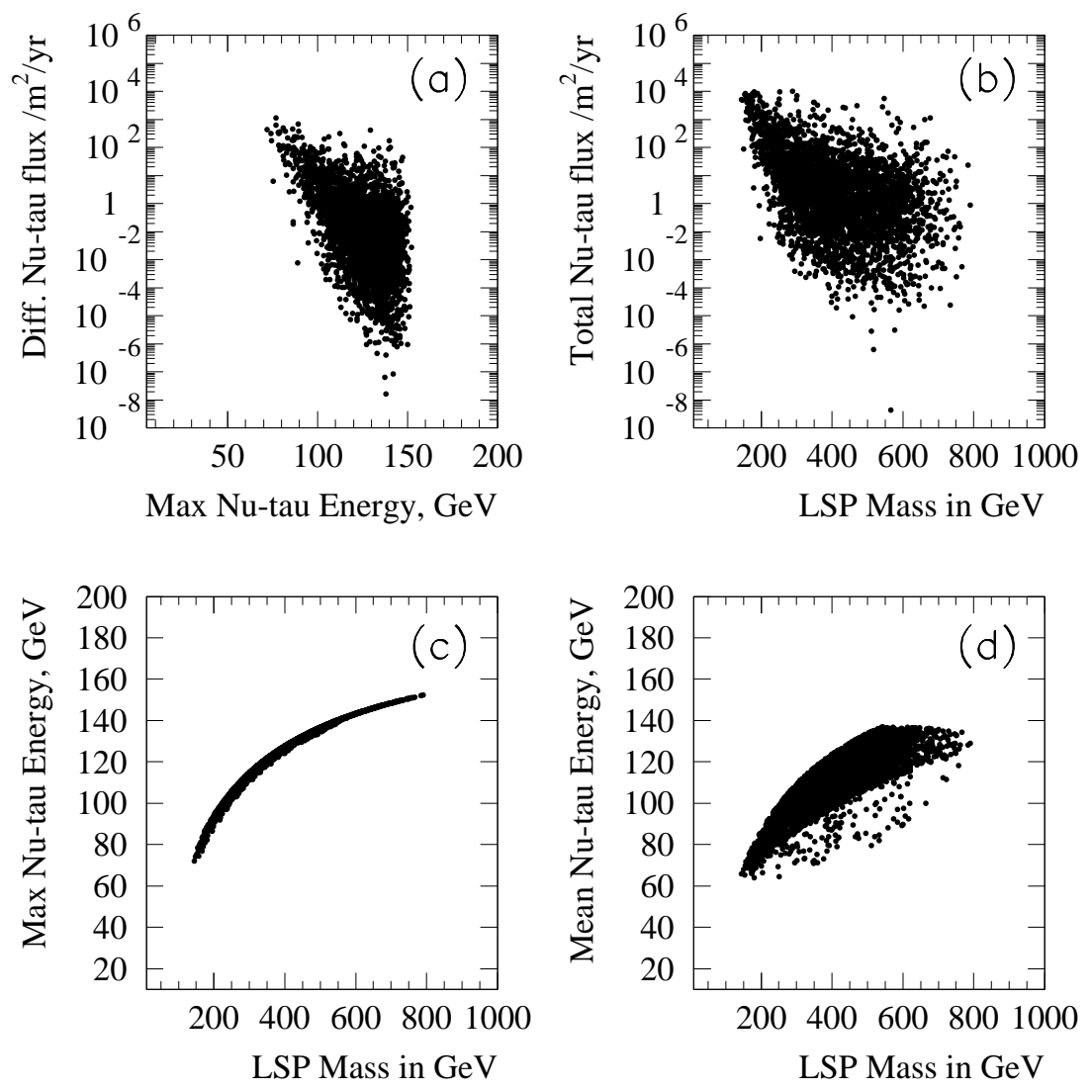

Fig. 4. (a) Differential flux of $\tau$ neutrinos from charged Higgs boson decay vs. maximum $\tau$ neutrino energy. (b) Total flux of $\tau$ neutrinos from charged Higgs bosons decay vs. neutralino mass $m_{\chi}$. (c) Maximum $\tau$ neutrino energy vs. neutralino mass $m_{\chi}$. (d) Mean $\tau$ neutrino energy vs. neutralino mass $m_{\chi}$. For the case of the Sun.

All other neutralino annihilation products can also produce $\tau$ neutrinos. But for our purpose only those channels which can produce $\tau$ neutrinos with comparable fluxes in the same energy range as the charged Higgs boson does should be taken into account. Therefore, the light fermion pairs need not be considered, since the annihilation of neutralino into them are negligible when the channel $\chi \chi \rightarrow H^{ \pm} W^{\mp}$ is open. The annihilation into top quark pair is one of the dominant channels for heavier neutralinos. Top quark decays almost exclusively via $t \rightarrow W^{+} b$ with energy $m_{\chi}$. We make an estimate using the formulae given in [9] and found that though the maximum $\tau$ neutrino energy from top quark decay is also roughly $m_{\chi}$, the differential flux at the maximum energy value is negligible. Furthermore, the energy spectrum is much softer. Therefore we do not take into account the contribution from top quark decay when considering energetic $\tau$ neutrinos from charged Higgs decay. Following similar arguments, 
the neutral Higgs bosons $h^{0}, H^{0}$ and $A^{0}$ are also excluded. The gauge bosons $W^{ \pm}$and $Z$ decay with energy $m_{\chi}$ into $\tau$ neutrinos with branching ratios 0.105 and 0.067 , respectively. For heavier neutralinos, the maximum energy of the $\tau$ neutrinos from gauge boson decay is also roughly $m_{\chi}$, the mean energy is always $m_{\chi} / 2$. Our calculations show that the $\tau$ neutrino flux from gauge boson decays is comparable to or even greater than that from charged Higgs decay in the same energy range. One can expect an enhancement of the total $\tau$ neutrino flux by a factor of 2 due to the contributions from gauge boson pairs from neutralino annihilation.

Therefore the $\nu_{\tau}$ signal from the charged Higgs bosons one can in principle find only searching for an enhancement of the $\nu_{\tau}$ spectrum at highest $\tau$ neutrino energies. The larger the LSP and the charged Higgs boson masses the stronger can be the expected enhancement.

\section{Conclusions}

In this work we studied the role of the Higgs bosons in the indirect detection of the WIMPs via their annihilation into energetic neutrinos. We performed our calculations on the basis of the MSSM parameter space at the electroweak scale, we did not assume any universality relations for the parameters at the unification scale.

First we have investigated the contribution to the total upward-going muon detection rate from neutrinos produced in the neutralino annihilation final states containing Higgs bosons. We found that their contribution is in general significant if the neutralino is heavier than about $150 \mathrm{GeV}$, and is becoming dominant in the case of the Sun as the current indirect WIMPs detection experiments reach the sensitivities of $\sim 10^{-3} \div 10^{-4} \mathrm{~m}^{-2} \mathrm{yr}^{-1}$. But most important, the final states containing Higgs bosons result in a lower bound on the expected detection rate from the Sun of $\sim 10^{-7} \div 10^{-8} \mathrm{~m}^{-2} \mathrm{yr}^{-1}$ for neutralinos heavier than about $200 \mathrm{GeV}$.

While the neutral Higgs bosons do not decay into muon neutrinos directly, the charged Higgs boson produced in the neutralino annihilation channel $\chi \chi \rightarrow$ $W^{ \pm} H^{\mp}$ has a large probability to decay into $\tau$ neutrinos directly via $H^{ \pm} \rightarrow$ $\tau \nu_{\tau}$. We expect them to be amongst the most energetic neutrinos from WIMPs annihilations and to have a very hard energy spectrum. We have estimated the energy and flux of these $\tau$ neutrinos from the Earth and the Sun, whereby in the case of the Sun the effects of neutrino stopping and absorption due to neutrino interactions with the solar medium were taken into account following [10]. Our results show that the maximum energy of the $\tau$ neutrinos from charged Higgs decay in the Earth is roughly the neutralino mass $m_{\chi}$, and their mean energy is just the energy of the charged Higgs boson, which depends only on the masses of the neutralino and the charged Higgs. The flux is in most cases below $1 \mathrm{~m}^{-2} \mathrm{yr}$

${ }^{-1}$ and lower for heavier neutralinos. Only for neutralino masses around 150-250 $\mathrm{GeV}$ one can expect higher $\tau$ neutrino flux. 
In the case of the Sun, the energies of the $\tau$ neutrinos are strongly degraded due the interactions with the solar medium (not greater than $150 \mathrm{GeV}$ even for the largest neutralino mass), the energy spectrum is also softer. But since all neutrinos propagating from the core of the Sun to the surface suffer from the same effects, the $\tau$ neutrinos from charged Higgs decay in the Sun are still among the most energetic and in principle can be separated when detected. Furthermore, one can expect much higher $\tau$ neutrino flux from the Sun, about $10^{2} \div 10^{4} \mathrm{~m}^{-2} \mathrm{yr}^{-1}$, than from the Earth.

The possible observation of such energetic $\tau$ neutrinos would be an evidence of the neutralino annihilation into charged Higgs bosons. In SUSY scenarios with very heavy sfermion and gaugino masses the existence of the charged Higgs boson could be considered as a clear signal of the supersymmetry.

\section{Acknowledgments}

We thank Prof. Pran Nath and Dr. O. V. Suvorova for the helpful discussions.

\section{References}

1. G. Jungman, M. Kamionkowski, K. Griest, Phys. Rept. 267 (1996) 195

2. O. Suvorova in Proceedings of the Second International Conference on Particle Physics Beyond the Standard Model, Castle Ringberg, Germany 1999, edited by H. V. Klapdor-Kleingrothaus and I. Krivosheina

3. V. Berezinsky, A. Bottino, J. Ellis, N. Fornengo, G. Mignola, S. Scopel, Astropart. Phys. 5 (1996) 333

4. L. Bergstrom, Preprint astro-ph/9902172

L. Bergstrom, J. Edsjo, P. Gondolo, Phys. Rev. D58 (1998) 103519

L. Bergstrom, J. Edsjo, P. Gondolo, Phys. Rev. D55 (1997) 1765

5. V. A. Bednyakov, H. V. Klapdor-Kleingrothaus, Phys. Rev. D62 (2000) 043524

6. J. F. Gunion, H. E. Haber, G. Kane and S. Dawson, The Higgs Hunter's Guide, Addison-Wesley 1990

7. A. Djouadi, J. Kalinowski, M. Spira, Comput. Phys. Commun. 108 (1998) 56

8. D. E. Groom et al. (Particle Data Group), Eur. Phys. Jour. C15 (2000) 1

9. G. Jungman, M. Kamionkowski, Phys. Rev. D51 (1995) 328

10. S. Ritz, D. Seckel, Nucl. Phys. B304 (1988) 877

11. M. Mori et al., Phys. Rev. D48 (1993) 5505 